Klüppelberg, Lindner, Maller:

Continuous time volatility modelling: COGARCH versus Ornstein-Uhlenbeck models

Sonderforschungsbereich 386, Paper 426 (2005)

Online unter: http://epub.ub.uni-muenchen.de/

Projektpartner
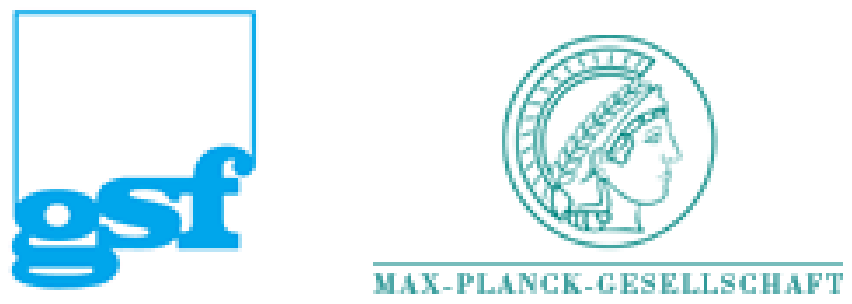


\title{
Continuous Time Volatility Modelling: COGARCH versus Ornstein-Uhlenbeck Models
}

\author{
Claudia Klüppelberg ${ }^{1}$, Alexander Lindner ${ }^{1}$, and Ross Maller $^{2}$ \\ 1 Centre for Mathematical Sciences, Munich University of Technology, D-85747 \\ Garching, Germany \{cklu, lindner\}@ma.tum.de \\ 2 Centre for Mathematical Analysis, and School of Finance \& Applied Statistics, \\ Australian National University, Canberra, ACT, Australia \\ Ross.Maller@anu .edu .au
}

Summary. We compare the probabilistic properties of the non-Gaussian OrnsteinUhlenbeck based stochastic volatility model of Barndorff-Nielsen and Shephard (2001) with those of the COGARCH process. The latter is a continuous time GARCH process introduced by the authors (2004). Many features are shown to be shared by both processes, but differences are pointed out as well. Furthermore, it is shown that the COGARCH process has Pareto like tails under weak regularity conditions.

Key words: COGARCH, continuous time GARCH, GARCH, generalised Ornstein-Uhlenbeck process, Lévy process, self-decomposable distribution, stochastic volatility model, tail behaviour

\section{Introduction}

It is common wisdom among financial researchers and the banking industry that volatility is stochastic, has jumps, and often exhibits long range dependence. Since such financial data as log-prices and exchange rates often come as high-frequency intra-day data, continuous time models are useful. There have been two main approaches.

The first, mathematical one is based on semimartingale (no arbitrage) theory, takes its starting point as the Black-Scholes model, and introduces a stochastic volatility process. For an introduction and overview of stochastic volatility models, we refer to Shephard [25]. The second, econometric, approach is based on empirical properties of financial time series. A recent model fitting into both these approaches and having received much attention is the stochastic volatility model of Barndorff-Nielsen and Shephard [2, 3, 4]. There, the volatility process is modelled as an Ornstein-Uhlenbeck (OU) type 
process driven by a Lévy process (or a superposition of such OU type processes), and thus can exhibit jumps. The price process is then obtained using an independent Brownian motion as driving noise.

The majority of the models arising from the econometric approach are in discrete time. In particular, GARCH models and their extensions have been in the limelight as appropriate models to capture certain empirical facts of the empirical volatility process; see Engle [13] for an overview on GARCH modelling. In this area, motivated again by the availability of high-frequency data and by the option pricing problem, classical diffusion limits have been used in a natural way to suggest continuous time limits; see, e.g., Nelson [23] and Duan [12].

Unfortunately, in these situations, the limiting models can lose certain essential properties of the discrete time GARCH models. Moreover, they can have distinctly different statistical properties. As has been shown recently by Wang [28], parameter estimation in the discrete time GARCH and the corresponding continuous time limit stochastic volatility model may yield different estimates. Thus the continuous time models are probabilistically and statistically different from their discrete time progenitors.

It is surprising and counter-intuitive that Nelson's diffusion limit of the GARCH process is driven by two independent Brownian motions, i.e. has two independent sources of randomness, whereas the discrete time GARCH process is driven only by a single white noise sequence. One of the features of the GARCH process is the idea that large innovations in the price process are almost immediately manifested as innovations in the volatility process, but this feedback mechanism is lost in models such as the Nelson continuous time version.

The phenomenon that a diffusion limit is driven by two independent Brownian motions, while the discrete time model is given in terms of a single white noise sequence, is not restricted to the classical GARCH process. Indeed, Duan [12] has shown that this occurs for many GARCH like processes. In this respect, Jeantheau [20] only recently developed a discrete time model having many features with the GARCH model in common, but having a diffusion limit driven by a single Brownian motion only.

In Klüppelberg, Lindner and Maller [22], the authors proposed a different approach to obtain a continuous time model. This "COGARCH" (continuous time GARCH) model, based on a single background driving Lévy process, is different from, though related to, other continuous time stochastic volatility models that have been proposed. It generalises the essential features of the discrete time GARCH process in a direct way.

It is natural to compare the two main approaches outlined above, i.e. stochastic volatility and GARCH type modelling. An empirical, likelihood inference based comparison between discrete time stochastic volatility and discrete time GARCH processes is given in Kim, Shephard and Chib [21]. In the present paper, we aim to compare the probabilistic properties of the COGARCH process with those of the stochastic volatility model of Barndorff- 
Nielsen and Shephard. It turns out that they share many mathematical properties, but that there are also certain differences. A striking difference is manifested in the behaviour (lightness or heaviness) of the tails of their onedimensional distributions. The stochastic volatility model can exhibit many different kinds of tail behaviour, depending on the driving Lévy process, whereas the COGARCH model has Pareto like (heavy) tails for essentially most driving Lévy processes.

The paper is structured as follows: in the next section, we recall the basic definitions of Lévy processes and give the definitions of the models under consideration. We then proceed to collect the properties of the models and compare them. The most obvious differences are pointed out in Section 2.3, while in Section 3 we consider properties of the process itself, such as strict stationarity, Markovian properties and pathwise behaviour. Then, in Section 4, second order properties are considered. It is shown that both processes have essentially the same kind of autocovariance structure. Section 5 focusses on distributional properties of both models. While it is well-known that the stationary distribution of the squared volatility of the OU type process of Barndorff-Nielsen and Shephard is self-decomposable, in Section 5.1 the same is shown to hold for the COGARCH volatility. Then, in Section 5.3, we prove some new results, showing that the COGARCH model has Pareto like tails under wide conditions. Finally, a short conclusion is given in Section 6 .

\section{Definition of the models}

Both the OU as well as the COGARCH model are driven by a Lévy process $L=\left(L_{t}\right)_{t \geq 0}$, assumed to be càdlàg and defined on a probability space with appropriate filtration, satisfying the "usual conditions", i.e. right-continuity and completeness. We recall some properties of Lévy processes, see Bertoin [6] and Sato [24]: for each $t \geq 0$ the characteristic function of $L_{t}$ at $\theta \in \mathbb{R}$ can be written in the form

$$
\begin{aligned}
& E\left(\mathrm{e}^{\mathrm{i} \theta L_{t}}\right) \\
& =\exp \left(t\left(\mathrm{i} \gamma_{L} \theta-\tau_{L}^{2} \frac{\theta^{2}}{2}+\int_{-\infty}^{\infty}\left(\mathrm{e}^{\mathrm{i} \theta x}-1-\mathrm{i} \theta x 1_{\{|x| \leq 1\}}\right) \Pi_{L}(\mathrm{~d} x)\right)\right) .
\end{aligned}
$$

The constants $\gamma_{L} \in \mathbb{R}, \tau_{L}^{2} \geq 0$ (Gaussian part) and the measure $\Pi_{L}$ on $\mathbb{R}$ form the characteristic triplet of $L$; the Lévy measure $\Pi_{L}$ is required to satisfy $\int_{\mathbb{R}} \min \left(1, x^{2}\right) \Pi_{L}(\mathrm{~d} x)<\infty$. If in addition $\int_{\mathbb{R}} \min (1,|x|) \Pi_{L}(\mathrm{~d} x)<\infty$, then $\gamma_{L, 0}:=\gamma_{L}-\int_{[-1,1]} x \Pi_{L}(\mathrm{~d} x)$ is called the drift of $L$. A Lévy process is of finite variation if and only if $\int_{\mathbb{R}} \min (1,|x|) \Pi_{L}(\mathrm{~d} x)<\infty$ and $\tau_{L}^{2}=0$. In that case, the sample paths of $\left(L_{t}\right)_{t \geq 0}$ have finite variation on compacts. A Lévy process with nondecreasing sample paths is called a subordinator. These are exactly the Lévy processes of finite variation with non-negative drift and having Lévy measure concentrated on $(0, \infty)$. In the following considerations, we will only 
be interested in the situation when the Lévy measure is non-trivial, i.e. we always assume that $\Pi_{L}$ is nonzero.

\subsection{The Barndorff-Nielsen and Shephard OU process}

The stochastic volatility model presented in $[2,3,4]$ specifies the volatility as an Ornstein-Uhlenbeck process, driven by a subordinator. More precisely, let $\left(L_{t}\right)_{t \geq 0}$ be a subordinator and $\alpha>0$. Then the volatility process $\left(\widetilde{\sigma}_{t}\right)_{t \geq 0}$ is defined by the stochastic differential equation (SDE)

$$
\mathrm{d} \widetilde{\sigma}_{t}^{2}=-\alpha \widetilde{\sigma}_{t}^{2} \mathrm{~d} t+\mathrm{d} L_{\alpha t}, \quad t \geq 0,
$$

where $\widetilde{\sigma}_{0}^{2}$ is a finite random variable independent of $\left(L_{t}\right)_{t \geq 0}$ and $\widetilde{\sigma}_{t}:=\sqrt{\widetilde{\sigma}_{t}^{2}}$. The solution to (2) is the Ornstein-Uhlenbeck type process ("OU process")

$$
\tilde{\sigma}_{t}^{2}=\left(\int_{0}^{t} \mathrm{e}^{\alpha s} \mathrm{~d} L_{\alpha s}+\widetilde{\sigma}_{0}^{2}\right) \mathrm{e}^{-\alpha t}, \quad t \geq 0 .
$$

The (logarithmic) price process $\left(\widetilde{G}_{t}\right)_{t \geq 0}$ is then modelled by the SDE

$$
\mathrm{d} \widetilde{G}_{t}=\left(\mu+b \widetilde{\sigma}_{t}^{2}\right) \mathrm{d} t+\widetilde{\sigma}_{t} \mathrm{~d} W_{t}, \quad t \geq 0, \quad \widetilde{G}_{0}=0,
$$

where $\mu$ and $b$ are constants and $\left(W_{t}\right)_{t \geq 0}$ is standard Brownian motion, independent of $\widetilde{\sigma}_{0}^{2}$ and the Lévy process $\left(L_{t}\right)_{t \geq 0}$. The Itô solution of this SDE is given by

$$
\widetilde{G}_{t}=\mu t+b \int_{0}^{t} \widetilde{\sigma}_{s}^{2} \mathrm{~d} s+\int_{0}^{t} \widetilde{\sigma}_{s} \mathrm{~d} W_{s}, \quad t \geq 0 .
$$

The logarithmic asset returns over time periods of length $r>0$ are then given by $\widetilde{G}_{t}^{(r)}:=\widetilde{G}_{t+r}-\widetilde{G}_{t}, t \geq 0$. In the following, the notation $\widetilde{G}_{t}$ and $\widetilde{\sigma}_{t}$ (with tildes) will always refer to the processes of Barndorff-Nielsen and Shephard just defined. In contrast, the COGARCH process defined below will always be denoted by $G_{t}$ with volatility $\sigma_{t}$ (without tildes). If the driving Lévy process $\left(L_{t}\right)_{t \geq 0}$ refers to the OU process, then it will always be assumed to be a subordinator.

\subsection{The COGARCH $(1,1)$ model}

The COGARCH$(1,1)$ process (see [22]) is motivated by the discrete time $\operatorname{GARCH}(1,1)$ process $\left(Y_{n}\right)_{n \in \mathbb{N}_{0}}$, satisfying

$$
Y_{n}=\varepsilon_{n} \sigma_{n, \mathrm{disc}}, \quad \text { where } \quad \sigma_{n, \mathrm{disc}}^{2}=\beta+\lambda Y_{n-1}^{2}+\delta \sigma_{n-1, \mathrm{disc}}^{2}, \quad n \in \mathbb{N},
$$

$\sigma_{n, \text { disc }}:=\sqrt{\sigma_{n, \text { disc }}^{2}}$, and $\left(\varepsilon_{n}\right)_{n \in \mathbb{N}_{0}}$ is a sequence of independent and identically distributed random variables, independent of $\sigma_{0, \text { disc }}^{2}$. Here, $\mathbb{N}=\{1,2,3, \ldots\}$ 
denotes the set of positive integers and $\mathbb{N}_{0}=\mathbb{N} \cup\{0\}$. The recursion in (5) can be solved to give

$$
\begin{aligned}
& \sigma_{n, \mathrm{disc}}^{2} \\
& =\left(\beta \int_{0}^{n} \exp \left\{-\sum_{j=0}^{\lfloor s\rfloor} \log \left(\delta+\lambda \varepsilon_{j}^{2}\right)\right\} \mathrm{d} s+\sigma_{0, \mathrm{disc}}^{2}\right) \exp \left\{\sum_{j=0}^{n-1} \log \left(\delta+\lambda \varepsilon_{j}^{2}\right)\right\} .
\end{aligned}
$$

To define the continuous time version, the innovations $\varepsilon_{n}$ are replaced by the jumps of a Lévy process. Let $\left(L_{t}\right)_{t \geq 0}$ be a Lévy process with jumps $\Delta L_{t}=$ $L_{t}-L_{t-}, t \geq 0$, and let $0<\delta<1, \bar{\lambda} \geq 0$. Define a càdlàg process $\left(X_{t}\right)_{t \geq 0}$ by

$$
X_{t}=-t \log \delta-\sum_{0<s \leq t} \log \left(1+(\lambda / \delta)\left(\Delta L_{s}\right)^{2}\right), \quad t \geq 0 .
$$

Then, with $\beta>0$ and $\sigma_{0}^{2}$ a finite random variable, independent of $\left(L_{t}\right)_{t \geq 0}$, define the (left-continuous) volatility process $\left(\sigma_{t}\right)_{t \geq 0}$ by

$$
\sigma_{t}^{2}=\left(\beta \int_{0}^{t} \mathrm{e}^{X_{s}} \mathrm{~d} s+\sigma_{0}^{2}\right) \mathrm{e}^{-X_{t-}}, \quad t \geq 0,
$$

where $\sigma_{t}:=\sqrt{\sigma_{t}^{2}}$, and define the integrated continuous time GARCH process ("COGARCH") $\left(G_{t}\right)_{t \geq 0}$ as the càdlàg process satisfying

$$
\mathrm{d} G_{t}=\sigma_{t} \mathrm{~d} L_{t}, \quad t \geq 0, \quad G_{0}=0 .
$$

Thus $G$ jumps at the same times as $L$ does, and has jumps of size $\Delta G_{t}=$ $\sigma_{t} \Delta L_{t}, t \geq 0$. The logarithmic asset returns over time periods of length $r>0$ are then modelled by $G_{t}^{(r)}:=G_{t+r}-G_{t}, t \geq 0$.

In [22], Proposition 3.1, it is shown that the process $\left(X_{t}\right)_{t \geq 0}$ is itself a spectrally negative Lévy process of finite variation, with drift $\gamma_{X, 0}=-\log \delta$ and zero Gaussian component $\tau_{X}^{2}=0$. The Lévy measure $\Pi_{X}$ is the image measure of $\Pi_{L}$ under the transformation $\mathbb{R} \rightarrow(-\infty, 0], x \mapsto-\log \left(1+(\lambda / \delta) x^{2}\right)$.

\subsection{A first comparison}

Despite their arising and being motivated in quite different ways, the volatility processes $\sigma^{2}$ and $\widetilde{\sigma}^{2}$ are strikingly analogous in satisfying the general OrnsteinUhlenbeck equations (3) and (7). But an obvious difference between the price processes is that the OU process of Barndorff-Nielsen and Shephard is fed into a Hull-White model, driven by an independent Brownian motion, whereas the COGARCH price process is driven by the same Lévy process as is used in the volatility. Furthermore, the SDE defining $\widetilde{G}_{t}$ has an additional drift term $\left(\mu+b \widetilde{\sigma}_{t}^{2}\right) \mathrm{d} t$, which does not occur in (8). It is possible to add such a drift term to (8) as well, but we will not do this since there is already a correspondence 
of $G$ to the discrete time GARCH process without the necessity for an extra drift term.

Another obvious difference concerns the sample path properties of the price processes: $\left(\widetilde{G}_{t}\right)_{t \geq 0}$ will have continuous sample paths, inherited from the driving Brownian motion (see e.g. Jacod and Shiryaev [19]), while $\left(G_{t}\right)_{t \geq 0}$ exhibits jumps. Both these factors can be useful in different ways in practice.

For the volatility processes, note that both $\left(\widetilde{\sigma}_{t}^{2}\right)_{t \geq 0}$ and $\left(\sigma_{t}^{2}\right)_{t \geq 0}$ exhibit jumps. While $\left(\widetilde{\sigma}_{t}\right)_{t \geq 0}$ is right-continuous, $\left(\sigma_{t}\right)_{t \geq 0}$ is left-continuous. This is a minor difference, since $\widetilde{G}_{t}$ is driven by Brownian motion, and hence $\widetilde{\sigma}_{t}$ in (4) could equally well be replaced by $\widetilde{\sigma}_{t-}$. A more striking difference between the volatility processes is that in (3) the driving Lévy process of the volatility is in the integrator, while in (7) it appears in the integrand. Despite these facts, we will see that both volatility processes nevertheless share many common features.

\section{Properties of the processes}

In this section we shall consider Markov and stationarity properties, link the integrated squared volatility and the quadratic variation for both processes, and exhibit some pathwise properties of the volatility processes. We start by mentioning that not only does $\widetilde{\sigma}_{t}$ satisfy a SDE, but so does $\sigma_{t}$, see Proposition 3.1 below, which was proved in [22], Proposition 3.2.

Proposition 1. [SDE and solution for $\sigma$ ]

The squared volatility process $\left(\sigma_{t}^{2}\right)_{t \geq 0}$ of the COGARCH process satisfies the stochastic differential equation

$$
\mathrm{d} \sigma_{t+}^{2}=\beta \mathrm{d} t+\sigma_{t}^{2} \mathrm{e}^{X_{t-}} \mathrm{d}\left(\mathrm{e}^{-X_{t}}\right), \quad t>0,
$$

and we have

$$
\sigma_{t}^{2}=\beta t+\log \delta \int_{0}^{t} \sigma_{s}^{2} \mathrm{~d} s+(\lambda / \delta) \sum_{0<s<t} \sigma_{s}^{2}\left(\Delta L_{s}\right)^{2}+\sigma_{0}^{2}, \quad t \geq 0 .
$$

Both volatility processes are Markovian:

Theorem 1. [Markov properties of the processes]

Both the squared volatility processes $\left(\widetilde{\sigma}_{t}^{2}\right)_{t \geq 0}$ and $\left(\sigma_{t}^{2}\right)_{t \geq 0}$, as given by (3) and (7), respectively, are time-homogeneous Markov processes. Furthermore, the bivariate processes $\left(\widetilde{\sigma}_{t}, \widetilde{G}_{t}\right)_{t \geq 0}$ and $\left(\sigma_{t}, G_{t}\right)_{t \geq 0}$ are time-homogeneous Markov processes.

Proof. For the fact that $\left(\widetilde{\sigma}_{t}^{2}\right)_{t \geq 0}$ is a time homogeneous Markov process if $\alpha=1$ see Sato [24], Lemma 17.1 and its preceding discussion. For general $\alpha>0$, the assertions on $\left(\widetilde{\sigma}_{t}^{2}\right)_{t \geq 0}$ and $\left(\widetilde{\sigma}_{t}, \widetilde{G}_{t}\right)_{t \geq 0}$ can be seen as follows. We have 


$$
\tilde{\sigma}_{t}^{2}=\widetilde{\sigma}_{y}^{2} \mathrm{e}^{\alpha(y-t)}+\int_{y}^{t} \mathrm{e}^{\alpha(s-t)} \mathrm{d} L_{\alpha s}=\mathrm{e}^{\alpha(y-t)}\left(\tilde{\sigma}_{y}^{2}+\int_{0}^{\alpha(t-y)} \mathrm{e}^{v} \mathrm{~d} L_{v+\alpha y}\right) .
$$

Since $\left\{L_{\alpha s}\right\}_{y \leq s \leq t}$ is independent of the $\sigma$-algebra generated by $\left(\widetilde{\sigma}_{u}^{2}\right)_{0 \leq u \leq y}$, the first equation gives the Markov property for $\widetilde{\sigma}_{t}$, and since the distribution of the expression on the righthand side depends only on $t-y$ we see that $\widetilde{\sigma}^{2}$ is time homogeneous. The Markovian property of $\left(\widetilde{\sigma}_{t}, \widetilde{G}_{t}\right)_{t \geq 0}$ follows from

$$
\widetilde{G}_{t}=\widetilde{G}_{y}+\mu(t-y)+b \int_{y}^{t} \widetilde{\sigma}_{s}^{2} \mathrm{~d} s+\int_{y}^{t} \widetilde{\sigma}_{s} \mathrm{~d} W_{s}, 0 \leq y<t .
$$

For the corresponding results on $\left(\sigma_{t}^{2}\right)_{t \geq 0}$ and $\left(\sigma_{t}, G_{t}\right)_{t \geq 0}$, see [22], Theorem 3.2 and Corollary 3.1.

The Markov property of the squared volatility processes can be regarded as a special case of a result on more general Ornstein-Uhlenbeck processes. Carmona, Petit and Yor [10] consider processes of the form

$$
V_{t}=\mathrm{e}^{\xi_{t}}\left(\int_{0}^{t} \mathrm{e}^{-\xi_{s-}} \mathrm{d} \eta_{s}+V_{0}\right), \quad t \geq 0,
$$

where $\left(\xi_{t}, \eta_{t}\right)_{t \geq 0}$ is a two-dimensional Lévy process, independent of $V_{0}$. Then $\left(V_{t}\right)_{t \geq 0}$ is a time homogeneous Markov process, see [10], Corollary 5.2. If $\left(\xi_{t}\right)_{t \geq 0}$ and $\left(\eta_{t}\right)_{t \geq 0}$ are independent, then [10] shows that $V_{t} \stackrel{D}{=} \int_{0}^{t} \mathrm{e}^{\xi_{s-}} \mathrm{d} \eta_{s}+$ $V_{0} \mathrm{e}^{\xi_{t}}$. (Throughout, "D=" means "equal in distribution".) Without assuming independence of $\xi$ and $\eta$, Erickson and Maller [16], Theorem 2, give necessary and sufficient conditions for the a.s. existence of the integral $\int_{0}^{\infty} \mathrm{e}^{\xi_{t-}} \mathrm{d} \eta_{t}$. When this occurs and $\xi$ and $\eta$ are independent, there is a stationary solution, $V_{\infty}$, say, and $V_{t}$ converges in distribution to this as $t \rightarrow \infty$ (see Carmona et al. [11], Theorem 3.1 and its proof). Theorem 2 below can be deduced from these results. (We remark that separate proofs for the two types of volatility process can be given without appealing to properties of the generalised OUprocess $\left(V_{t}\right)_{t \geq 0}$. For $\left(\widetilde{\sigma}_{t}^{2}\right)_{t \geq 0}$, see $[2,3]$ or Sato [24], Theorems 17.5, 17.11 and Corollary 17.9 (apart from part (c) below), while for $\left(\sigma_{t}^{2}\right)_{t \geq 0}$ see [22], Theorems 3.1, 3.2 and Corollary 3.1.)

Theorem 2. [Stationarity condition for $\widetilde{\sigma}$ and $\sigma$ ]

(a) The squared volatility process $\left(\widetilde{\sigma}_{t}^{2}\right)_{t \geq 0}$ of the $O U$ model converges in distribution to a finite random variable $\widetilde{\sigma}_{\infty}^{2}$ as $t \rightarrow \infty$ if and only if

$$
\int_{1}^{\infty} \log (y) \Pi_{L}(\mathrm{~d} y)<\infty
$$

In that case,

$$
\widetilde{\sigma}_{\infty}^{2} \stackrel{D}{=} \int_{0}^{\infty} \mathrm{e}^{-s} \mathrm{~d} L_{s} .
$$


(b) The squared volatility process $\left(\sigma_{t}^{2}\right)_{t \geq 0}$ of the COGARCH model converges in distribution to a finite random variable $\sigma_{\infty}^{2}$ as $t \rightarrow \infty$ if and only if

$$
\int_{\mathbb{R}} \log \left(1+(\lambda / \delta) y^{2}\right) \Pi_{L}(\mathrm{~d} y)<-\log \delta
$$

(which, since $\delta>0$, incorporates the requirement that the integral be finite), in which case

$$
\sigma_{\infty}^{2} \stackrel{D}{=} \beta \int_{0}^{\infty} \mathrm{e}^{-X_{t}} \mathrm{~d} t
$$

(c) If (10) or (12) are not satisfied, respectively, then the squared volatility process diverges in probability to $\infty$ as $t \rightarrow \infty$.

(d) A stationary solution of $\left(\widetilde{\sigma}_{t}^{2}\right)_{t \geq 0}$ or $\left(\sigma_{t}^{2}\right)_{t \geq 0}$ exists if and only if (10) or (12) are satisfied, in which case the stationary distribution at time $t$ is the distribution of $\widetilde{\sigma}_{\infty}^{2}$ or $\sigma_{\infty}^{2}$, respectively. In that case, $\left(\widetilde{G}_{t}\right)_{t \geq 0}$ and $\left(G_{t}\right)_{t \geq 0}$ have stationary increments, i.e. the increment processes $\left(\widetilde{G}_{t}^{(r)}\right)_{t \geq 0}$ and $\left(G_{t}^{(r)}\right)_{t \geq 0}$ are stationary for each fixed $r>0$.

It is interesting to observe that the stationarity condition for $\left(\widetilde{\sigma}_{t}^{2}\right)_{t \geq 0}$ and the distribution of $\widetilde{\sigma}_{\infty}^{2}$ depend on the Lévy measure $\Pi_{L}$ only, whereas (12) and $\sigma_{\infty}^{2}$ depend on $\Pi_{L}$ and on the parameters $\delta$ and $\lambda$. For the OU model, this is a consequence of the unusual timing $\mathrm{d} L_{\alpha t}$ in (2), chosen deliberately by Barndorff-Nielsen and Shephard [3] to separate the stationary distribution from the dynamical structure, which depends on $\alpha$.

Next we investigate pathwise properties of the volatility processes, especially the behaviour between jumps if the driving Lévy process is compound Poisson.

Proposition 2. [Pathwise behaviour of $\widetilde{\sigma}$ and $\sigma$ ] (a) The volatility $\sigma_{t}$ at time $t$ of the GOGARCH process satisfies

$$
\sigma_{t}^{2} \geq \frac{\beta}{-\log \delta}\left(1-\mathrm{e}^{t \log \delta}\right), \text { for all } t \geq 0 .
$$

If $\sigma_{t_{0}}^{2} \geq \frac{\beta}{-\log \delta}$ for some $t_{0}$, then $\sigma_{t}^{2} \geq \frac{\beta}{-\log \delta}$ for every $t \geq t_{0}$.

If $\sigma_{t}^{2} \stackrel{D}{=} \sigma_{\infty}^{2}$ is the stationary version, then

$$
\sigma_{\infty}^{2} \geq \frac{\beta}{-\log \delta} \quad \text { a.s. }
$$

The stationary version $\widetilde{\sigma}_{\infty}^{2}$ of the OU-process is bounded from below (i.e. bounded away from 0 ) if and only if the drift term $\gamma_{L, 0}$ of the subordinator $\left(L_{t}\right)_{t \geq 0}$ is strictly positive.

(b) The jumps of both squared volatility processes at time $t>0$ are described by

$$
\widetilde{\sigma}_{t}^{2}-\widetilde{\sigma}_{t-}^{2}=\Delta L_{\alpha t}, \quad \sigma_{t+}^{2}-\sigma_{t}^{2}=(\lambda / \delta) \sigma_{t}^{2}\left(\Delta L_{t}\right)^{2} .
$$


(c) Let $\left(L_{t}\right)_{t \geq 0}$ be a compound Poisson process with jump times $0=T_{0}<$ $T_{1}<\ldots$ Then the OU volatility satisfies for $t \in\left(T_{j} / \alpha, T_{j+1} / \alpha\right), j \in \mathbb{N}_{0}$,

$$
\frac{\mathrm{d}}{\mathrm{d} t} \widetilde{\sigma}_{t}^{2}=-\alpha \widetilde{\sigma}_{t}^{2}, \quad \widetilde{\sigma}_{t}^{2}=\widetilde{\sigma}_{T_{j} / \alpha}^{2} \mathrm{e}^{-\left(\alpha t-T_{j}\right)},
$$

while the COGARCH volatility satisfies for $t \in\left(T_{j}, T_{j+1}\right)$,

$$
\frac{\mathrm{d}}{\mathrm{d} t} \sigma_{t}^{2}=\beta+(\log \delta) \sigma_{t}^{2}, \quad \sigma_{t}^{2}=\frac{\beta}{-\log \delta}+\left(\sigma_{T_{j}+}^{2}+\frac{\beta}{\log \delta}\right) \mathrm{e}^{\left(t-T_{j}\right) \log \delta} .
$$

Proof. (a) From (6) follows that for $0 \leq s<t$,

$$
X_{s}-X_{t-}=(t-s) \log \delta+\sum_{s<u<t} \log \left(1+(\lambda / \delta)\left(\Delta L_{u}\right)^{2}\right) \geq(t-s) \log \delta .
$$

In particular,

$$
\begin{aligned}
\sigma_{t}^{2} & =\beta \int_{0}^{t} \mathrm{e}^{X_{s}-X_{t-}} \mathrm{d} s+\sigma_{0}^{2} \mathrm{e}^{-X_{t-}} \\
& \geq \beta \int_{0}^{t} \mathrm{e}^{(t-s) \log \delta} \mathrm{d} s=\frac{\beta}{-\log \delta}\left(1-\mathrm{e}^{t \log \delta}\right) .
\end{aligned}
$$

Then (13) follows as $t \rightarrow \infty$. Now let $t>t_{0}$ and suppose that $\sigma_{t_{0}}^{2} \geq \frac{\beta}{-\log \delta}$. In equation (3.12) of [22] it was shown that

$$
\sigma_{t}^{2}=\mathrm{e}^{X_{t_{0}-}-X_{t-}} \sigma_{t_{0}}^{2}+\beta \int_{t_{0}}^{t} \mathrm{e}^{X_{s}-X_{t-}} \mathrm{d} s .
$$

From (14) then follows

$$
\begin{aligned}
\sigma_{t}^{2} & \geq \mathrm{e}^{\left(t-t_{0}\right) \log \delta} \sigma_{t_{0}}^{2}+\beta \int_{t_{0}}^{t} \mathrm{e}^{\left(s-t_{0}\right) \log \delta} \mathrm{d} s \\
& \geq \mathrm{e}^{\left(t-t_{0}\right) \log \delta}\left(\frac{\beta}{-\log \delta}\right)+\left(\frac{\beta}{-\log \delta}\right)\left(1-\mathrm{e}^{\left(t-t_{0}\right) \log \delta}\right)=\frac{\beta}{-\log \delta} .
\end{aligned}
$$

That $\widetilde{\sigma}_{\infty}^{2}$ is bounded from below if and only if the drift is non-zero follows from (11) and Sato [24], Example 17.10.

The proof of $(b)$ and $(c)$ follows easily from (3), (7) and (9).

Proposition 2 shows in particular that the stationary version of the COGARCH volatility process is always bounded away from 0 once $t>0$, which is not necessarily the case for the OU volatility. From $(b)$ it follows that if a volatility jump occurs for either process, then this jump is necessarily positive. For compound Poisson driving processes, between jumps the processes show similarities, since both decay exponentially (more precisely, the COGARCH process decays only once it rises above the lower bound $\beta /(-\log \delta)$, and before 
that it increases). However, note that $\left(\widetilde{\sigma}_{t}^{2}\right)$ satisfies a homogeneous differential equation, while $\left(\sigma_{t}^{2}\right)$ satisfies an inhomogeneous differential equation, between jumps.

Next, we link the integrated squared volatilities $\int_{0}^{t} \widetilde{\sigma}_{s}^{2} \mathrm{~d} s$ and $\int_{0}^{t} \sigma_{s}^{2} \mathrm{~d} s$ with the quadratic variations of the process $\widetilde{G}$ and $G$, respectively. For the definition and elementary properties of the quadratic variation $[Y, Y]_{t}$ of a semimartingale $\left(Y_{t}\right)_{t \geq 0}$, we refer to Jacod and Shiryaev [19], Chapter 1.

Proposition 3. [Quadratic variation and integrated squared volatility]

(a) For the stochastic volatility model of Barndorff-Nielsen and Shephard we have

$$
[\widetilde{G}, \widetilde{G}]_{t}=\int_{0}^{t} \widetilde{\sigma}_{s}^{2} \mathrm{~d} s, \quad t \geq 0 .
$$

(b) For the COGARCH model we have

$$
\frac{\lambda}{\delta}[G, G]_{t-}=\left(\frac{\lambda}{\delta} \tau_{L}^{2}-\log \delta\right) \int_{0}^{t} \sigma_{s}^{2} \mathrm{~d} s+\sigma_{t}^{2}-\sigma_{0}^{2}-\beta t, \quad t \geq 0 .
$$

Proof. (a) is clear from the general properties of stochastic integrals, see e.g. [19], while (b) follows from

$$
\begin{aligned}
{[G, G]_{t-} } & =\int_{0}^{t-} \sigma_{s}^{2} \mathrm{~d}[L, L]_{s} \\
& =\int_{0}^{t-} \sigma_{s}^{2} \mathrm{~d}\left(s \tau_{L}^{2}+\sum_{0<u \leq s}\left(\Delta L_{u}\right)^{2}\right)=\tau_{L}^{2} \int_{0}^{t} \sigma_{s}^{2} \mathrm{~d} s+\sum_{0<u<t} \sigma_{s}^{2}\left(\Delta L_{s}\right)^{2} .
\end{aligned}
$$

Plugging this into (9) gives (16).

The integrated quadratic variation is a key measure for stochastic volatility models. Its importance can be seen from equation (23) below. Now (15) means that the integrated volatility can be recovered from the quadratic variation. Equation (16) shows that for the COGARCH process, the integrated volatility can at least be expressed with the aid of the quadratic variation and the volatility at times $t$ and 0 by a reasonably simple formula. An expression in terms of the quadratic variation only cannot be expected, since the Lévy process in (8) has jumps.

\section{Second order properties}

In this section we shall concentrate on moments and autocorrelation functions of both the volatility processes and the price process. A short discussion of the cumulant transform for the OU process is included.

From now on, in order to avoid the trivial case of a deterministic volatility, we shall always assume $\lambda>0$ when dealing with the COGARCH process. 


\subsection{The volatility process}

In this section we derive moments and autocorrelation functions of the squared stochastic volatility processes $\left(\widetilde{\sigma}_{t}^{2}\right)_{t \geq 0}$ and $\left(\sigma_{t}^{2}\right)_{t \geq 0}$. For convenience we shall restrict ourselves to the case of the stationary versions of these volatility processes. We start with a preparatory lemma on exponential moments of $\left(X_{t}\right)_{t \geq 0}$ for the COGARCH volatility, which by (7) are related to moments of $\sigma_{t}^{2}$.

Lemma 1. [Exponential moments of $X$ ]

Let $X_{t}$ be given by (6), and keep $\kappa>0$ throughout.

(a) $E \mathrm{e}^{-\kappa X_{t}}<\infty$ for some $t>0$, or, equivalently, for all $t>0$, if and only if $E\left|L_{1}\right|^{2 \kappa}<\infty$.

(b) When $E \mathrm{e}^{-\kappa X_{1}}<\infty$, define $\Psi(\kappa)=\Psi_{X}(\kappa)=\log E \mathrm{e}^{-\kappa X_{1}}$. Then $|\Psi(\kappa)|<$ $\infty, E \mathrm{e}^{-\kappa X_{t}}=\mathrm{e}^{t \Psi(\kappa)}$, and

$$
\Psi(\kappa)=\kappa \log \delta+\int_{\mathbb{R}}\left(\left(1+(\lambda / \delta) y^{2}\right)^{\kappa}-1\right) \Pi_{L}(\mathrm{~d} y) .
$$

(c) If $\Psi(\kappa)<0$ for some $\kappa>0$, then $\Psi(d)<0$ for all $0<d<\kappa$.

(d) If $E\left|L_{1}\right|^{2 \kappa}<\infty$ and $\Psi(\kappa) \leq 0$ for some $\kappa>0$, then (12) holds, and a stationary version of $\left(\sigma_{t}^{2}\right)_{t \geq 0}$ exists.

Proof. $(a),(b)$ and $(c)$ are proved in Lemma 4.1 of [22]. For $(d)$, note that $\Psi(\kappa) \leq 0$ is equivalent to

$$
\frac{1}{\kappa} \int_{\mathbb{R}}\left(\left(1+\frac{\lambda}{\delta} y^{2}\right)^{\kappa}-1\right) \Pi_{L}(\mathrm{~d} y) \leq-\log \delta .
$$

Since $\log \left(1+(\lambda / \delta) y^{2}\right)<(1 / \kappa)\left(\left(1+(\lambda / \delta) y^{2}\right)^{\kappa}-1\right)$ for any $y \neq 0$ (as a consequence of $x>1+\log x$ for $x>1$ ), this implies (12).

Next we give conditions for the existence of moments of the squared volatility processes. For $\widetilde{\sigma}_{\infty}^{2}$ this is done in terms of the cumulants. Recall that the $\mathrm{cu}$ mulant transform of a random variable $Y$ is defined $\operatorname{asc}^{\operatorname{cum}_{Y}}(\theta):=\log E \mathrm{e}^{\mathrm{i} \theta Y}$, and that the $k$ th cumulant cum $_{Y, k}$ exists if and only if $E|Y|^{k}<\infty$, in which case it is given by

$$
\operatorname{cum}_{Y, k}:=\frac{1}{\mathrm{i}^{k}} \frac{\mathrm{d}^{k}}{\mathrm{~d} \theta^{k}} \operatorname{cum}_{Y}(0) .
$$

In particular,

$$
\operatorname{cum}_{Y, 1}=E Y, \quad \operatorname{cum}_{Y, 2}=\operatorname{Var}(Y) .
$$

Theorem 3. [Moments and ACF of $\widetilde{\sigma}$ and $\sigma$ ] Let $\widetilde{\sigma}_{\infty}^{2}$ and $\sigma_{\infty}^{2}$ have the stationary distributions of the volatility processes, respectively.

(a) The kth moment of $\widetilde{\sigma}_{\infty}^{2}$ is finite if and only if $E L_{1}^{k}<\infty, k \in \mathbb{N}$. In this case, the kth cumulants of $\widetilde{\sigma}_{\infty}^{2}$ and $L_{1}$ satisfy the relation 


$$
\operatorname{cum}_{\widetilde{\sigma}_{\infty}^{2}, k}=k^{-1} \operatorname{cum}_{L_{1}, k} .
$$

In particular, $E \widetilde{\sigma}_{\infty}^{2}=E L_{1}$, $\operatorname{Var}\left(\widetilde{\sigma}_{\infty}^{2}\right)=2^{-1} \operatorname{Var}\left(L_{1}\right)$. If $E L_{1}^{2}<\infty$, then the autocovariance function of the stationary squared volatility process satisfies

$$
\operatorname{Cov}\left(\tilde{\sigma}_{t}^{2}, \widetilde{\sigma}_{t+h}^{2}\right)=2^{-1} \operatorname{Var}\left(L_{1}\right) \mathrm{e}^{-\alpha h}, \quad t, h \geq 0 .
$$

(b) The $k$ th moment of $\sigma_{\infty}^{2}$ is finite if and only if $E L_{1}^{2 k}<\infty$ and $\Psi(k)<0$, $k \in \mathbb{N}$. In this case,

$$
E \sigma_{\infty}^{2 k}=k ! \beta^{k} \prod_{l=1}^{k} \frac{1}{-\Psi(l)}
$$

In particular, $E \sigma_{\infty}^{2}=\frac{\beta}{-\Psi(1)}, \operatorname{Var}\left(\sigma_{\infty}^{2}\right)=\beta^{2}\left(2 \Psi^{-1}(1) \Psi^{-1}(2)-\Psi^{-2}(1)\right)$. If $E L_{1}^{4}<\infty$ and $\Psi(2)<\infty$, then the autocovariance function of the stationary squared volatility process satisfies

$$
\operatorname{Cov}\left(\sigma_{t}^{2}, \sigma_{t+h}^{2}\right)=\beta^{2}\left(\frac{2}{\Psi(1) \Psi(2)}-\frac{1}{\Psi^{2}(1)}\right) \mathrm{e}^{-|\Psi(1)| h}, \quad t, h \geq 0 .
$$

Proof. (a) The existence of the moments of $\widetilde{\sigma}_{\infty}^{2}$ is a consequence of

$$
E L_{1}^{k} \leq \mathrm{e}^{k} E\left(\int_{0}^{1} \mathrm{e}^{-s} \mathrm{~d} L_{s}\right)^{k} \leq \mathrm{e}^{k} E\left(\int_{0}^{\infty} \mathrm{e}^{-s} \mathrm{~d} L_{s}\right)^{k}
$$

(recall that $L_{t}$ is a subordinator in the tilde setup) and

$$
\begin{aligned}
& E\left(\int_{0}^{\infty} \mathrm{e}^{-s} \mathrm{~d} L_{s}\right)^{k} \\
& \leq E\left(\sum_{i=0}^{\infty} \mathrm{e}^{-i}\left(L_{i+1}-L_{i}\right)\right)^{k} \\
& =\sum_{i_{1}=0}^{\infty} \cdots \sum_{i_{k}=0}^{\infty} \mathrm{e}^{-i_{1}-\ldots-i_{k}} E\left(\left(L_{i_{1}+1}-L_{i_{1}}\right) \cdots\left(L_{i_{k}+1}-L_{i_{k}}\right)\right)
\end{aligned}
$$

and the latter is finite if $E L_{1}^{k}<\infty$ by independence and identical distribution of the increments $L_{i_{j}+1}-L_{i_{j}}$. The relation between the cumulants (when they exist) and the formula for the autocovariance function can be found in [3], page 172 .

The proof of $(b)$ can be found in [22], Proposition 4.2 and Corollary 4.1. For (19), see also Carmona, Petit and Yor [10], Proposition 3.3.

Note that the moment condition $E L_{1}^{2 k}$ and $\Psi(k)<0$ for the COGARCH volatility already imply the existence of a stationary version by Lemma $1(\mathrm{~d})$. The same is true for the Ornstein-Uhlenbeck process, since $E L_{1}<\infty$ is equivalent to $\int_{1}^{\infty} x \Pi_{L}(\mathrm{~d} x)<\infty$, implying (10). 
It should be noted that, for $\widetilde{\sigma}_{\infty}^{2}$, the existence of moments depends only on the driving Lévy process $\left(L_{t}\right)_{t \geq 0}$, while for $\sigma_{\infty}^{2}$ it depends on the driving Lévy process as well as on the parameters. This is highlighted in the following Proposition, see [22], Proposition 4.3.

Proposition 4. [Dependence on parameters for moments of $\sigma$ ]

(a) For any Lévy process $\left(L_{t}\right)_{t \geq 0}$ with nonzero Lévy measure such that $\int_{\mathbb{R}} \log \left(1+y^{2}\right) \Pi_{L}(\mathrm{~d} y)$ is finite, there exist parameters $\delta, \lambda \in(0,1)$ for which $\sigma_{\infty}^{2}$ exists, but $E \sigma_{\infty}^{2}=\infty$.

(b) For any Lévy process $\left(L_{t}\right)_{t \geq 0}$ such that $E L_{1}^{2 k}<\infty(k \in \mathbb{N})$ and for any $\delta \in(0,1)$ there exists $\lambda_{\delta}>0$ such that the limit variable $\sigma_{\infty}^{2}$ exists with $E \sigma_{\infty}^{2 k}<\infty$ for any pair of parameters $(\delta, \lambda)$ such that $0<\lambda \leq \lambda_{\delta}$.

(c) Suppose $0<\delta<1, \lambda>0$. Then for no Lévy process $\left(L_{t}\right)_{t \geq 0}$ (with nonzero Lévy measure) do the moments of all orders of $\sigma_{\infty}^{2}$ exist. In particular, the Laplace transform $\mathrm{Ee}^{-\theta \sigma_{\infty}^{2}}$ of $\sigma_{\infty}^{2}$ does not exist for any $\theta<0$.

Much of the analysis in [3] is based on the connection between the cumulant functions of $L_{1}$ and $\widetilde{\sigma}_{\infty}^{2}$. In [1], page 178, it is shown that

$$
\operatorname{cum}_{\widetilde{\sigma}_{\infty}^{2}}(\theta)=\int_{0}^{\infty} \operatorname{cum}_{L_{1}}\left(e^{-s} \theta\right) \mathrm{d} s, \quad \operatorname{cum}_{L_{1}}(\theta)=\theta \frac{\mathrm{d}}{\mathrm{d} \theta} \operatorname{cum}_{\widetilde{\sigma}_{\infty}^{2}}(\theta)
$$

(provided they exist), see also [5], page 282, where a similar relation for the logarithms of the Laplace transforms is established. In contrast, for the COGARCH volatility, a feasible expression for the cumulant transform or the Laplace transform does not seem to be at hand. By Proposition 4, the Laplace transform of $\sigma_{\infty}^{2}$ does not exist in a (two-sided) neighbourhood of the origin. However, the Laplace transform of the random variable $\sigma_{\infty}^{-2}$ exists in a neighbourhood of the origin and $\sigma_{\infty}^{2}$ is determined by all its negative integer moments. This was shown by Bertoin and Yor [7], Proposition 2, who also give an expression for the negative integer moments.

\subsection{The price process}

In this section we investigate second order properties of the increments of the price processes $\left(\widetilde{G}_{t}\right)_{t \geq 0}$ and $\left(G_{t}\right)_{t \geq 0}$. From Section 2 recall the notation

$$
\widetilde{G}_{t}^{(r)}:=\widetilde{G}_{t+r}-\widetilde{G}_{t}, \quad G_{t}^{(r)}:=G_{t+r}-G_{t}, \quad t \geq 0, \quad r>0,
$$

corresponding to logarithmic asset returns over time periods of length $r$. We will work with the stationary version of the volatility process. By Theorem 2 this implies strict stationarity of the processes $\left(\widetilde{G}_{t}^{(r)}\right)_{t \geq 0}$ and $\left(G_{t}^{(r)}\right)_{t \geq 0}$, respectively. 
Theorem 4. [ACF of the price process]

Let $r>0$ be a fixed constant, and let $t \geq 0$.

(a) Let the price process $\left(\widetilde{G}_{t}\right)_{t>0}$ be defined by (4) for the stationary volatility process $\left(\widetilde{\sigma}_{t}\right)_{t \geq 0}$. Assume that $E L_{1}^{2}<\infty$. Then

$$
\begin{aligned}
E\left(\widetilde{G}_{t}^{(r)}\right) & =\left(\mu+b E L_{1}\right) r, \\
\operatorname{Var}\left(\widetilde{G}_{t}^{(r)}\right) & =r E L_{1}+b^{2} \operatorname{Var}\left(L_{1}\right)\left(r / \alpha-\left(1-\mathrm{e}^{-\alpha r}\right) / \alpha^{2}\right) .
\end{aligned}
$$

If $\mu=b=0$, then

$$
\operatorname{Cov}\left(\widetilde{G}_{t}^{(r)}, \widetilde{G}_{t+h}^{(r)}\right)=0
$$

for any $h \geq r$. If additionally $E L_{1}^{4}<\infty$, then there is a strictly positive constant $\widetilde{C}_{r}$ (not depending on $t$ ) such that

$$
\operatorname{Cov}\left(\left(\widetilde{G}_{t}^{(r)}\right)^{2},\left(\widetilde{G}_{t+h}^{(r)}\right)^{2}\right)=\widetilde{C}_{r} \mathrm{e}^{-\alpha h} \quad \forall h \in r \mathbb{N} .
$$

(b) Let the COGARCH process $\left(G_{t}\right)_{t \geq 0}$ be defined by (8) for the stationary volatility process $\left(\sigma_{t}\right)_{t>0}$. Suppose $\left(L_{t}\right)_{t>0}$ is a quadratic pure jump process (i.e. $\tau_{L}^{2}=0$ in (1)) with $E L_{1}^{2}<\infty, E L_{1}=0$, and that $\Psi(1)<0$. Then for any $h \geq r>0$,

$$
\begin{aligned}
E\left(G_{t}^{(r)}\right) & =0, \\
E\left(G_{t}^{(r)}\right)^{2} & =\frac{\beta r}{-\Psi(1)} E L_{1}^{2}, \\
\operatorname{Cov}\left(G_{t}^{(r)}, G_{t+h}^{(r)}\right) & =0 .
\end{aligned}
$$

Assume further that $E L_{1}^{4}<\infty$ and $\Psi(2)<0$. Then there is a non-negative constant $C_{r}$ (not depending on $t$ ) such that

$$
\operatorname{Cov}\left(\left(G_{t}^{(r)}\right)^{2},\left(G_{t+h}^{(r)}\right)^{2}\right)=C_{r} \mathrm{e}^{-|\Psi(1)| h} \quad \forall h \geq r .
$$

Assume further that $E L_{1}^{8}<\infty, \psi(4)<0$, that $\left(L_{t}\right)_{t \geq 0}$ is of finite variation and that $\int_{\mathbb{R}} x^{3} \Pi_{L}(\mathrm{~d} x)=0$. Then $C_{r}$ is strictly positive.

The proof of $(a)$ can be found in Section 4 of [3], while the proof of $(b)$ is given in [22], Proposition 5.1.

Theorem 4 tells us that for both models the returns are uncorrelated, while the squared returns are correlated. This agrees very much with empirical findings. In both models, the autocorrelation function of the squared returns decreases exponentially. Furthermore, we see that $\operatorname{Var}\left(G_{t}^{(r)}\right)$ is linear in $r$, while $\operatorname{Var}\left(\widetilde{G}_{t}^{(r)}\right)$ is asymptotically (affine) linear in $r$ as $r$ approaches 0 or $\infty$ (however, with different slopes for $r \rightarrow 0$ and $r \rightarrow \infty$ ).

\section{Distributional properties of the models}

In this section we investigate further properties of the stationary distribution of the volatility processes and the price processes. 


\subsection{Self-decomposability}

The distribution of a random variable $Y$ is called self-decomposable if for any $c \in(0,1)$ there exists a random variable $Z_{c}$, independent of $Y$, such that

$$
Y \stackrel{D}{=} c Y+Z_{c}
$$

Every self-decomposable distribution is infinitely divisible, and an infinitely divisible distribution is self-decomposable if and only if its Lévy measure has a Lévy density $w$, which can be represented as

$$
w(x)=\frac{k_{+}(x)}{x} 1_{x>0}+\frac{k_{-}(|x|)}{|x|} 1_{x<0}, \quad x \in \mathbb{R},
$$

where $k_{+}$and $k_{-}$are non-increasing non-negative functions on $(0, \infty)$. Not only has the Lévy measure a density, but also the distribution itself has. See Sato [24], Theorem 27.13, and Sections 15-17 there for examples and properties of self-decomposable distributions. As a further example, the class of generalised inverse Gaussian distributions is considered in [3].

The stationary distributions $\tilde{\sigma}_{\infty}^{2}$ of the Ornstein-Uhlenbeck model of Barndorff-Nielsen and Shephard [3] now have the nice property that they are self-decomposable. Furthermore, as $L$ varies over all subordinators, they constitute the class of all possible self-decomposable distributions whose support is contained in $[0, \infty)$, see Sato [24], Example 17.10 and Theorem 24.10. The correspondence between the Lévy density $w$ of $\widetilde{\sigma}_{\infty}^{2}$ and the Lévy measure $\Pi_{L}$ of the driving Lévy process $\left(L_{t}\right)_{t \geq 0}$ is given by

$$
w(x)=x^{-1} \Pi_{L}((x, \infty)), \quad x>0,
$$

see [4], equation (4.17). Interestingly, the stationary distribution $\sigma_{\infty}^{2}$ of the COGARCH process is self-decomposable, too. This was communicated to us by Samorodnitsky [27], who more generally showed that $\int_{0}^{\infty} e^{-X_{t}} \mathrm{~d} t$ is selfdecomposable for any spectrally negative Lévy process $\left(X_{t}\right)_{t \geq 0}$ such that $X_{t} \rightarrow+\infty$ a.s. We state this as a Theorem, and include Samorodnitsky's proof.

Theorem 5. The stationary distributions $\widetilde{\sigma}_{\infty}^{2}$ and $\sigma_{\infty}^{2}$ of both the squared volatility processes of the $\mathrm{OU}$-process and the COGARCH process are selfdecomposable.

Proof. We only need to show the result for $\sigma_{\infty}^{2}$. The process $\left(X_{t}\right)_{t \geq 0}$ defined in (6) is spectrally negative. Further, $X_{t} \rightarrow+\infty$ a.s. as $t \rightarrow \infty$ as a consequence of (12) (see [22], proof of Theorem 3.1). From this follows that the stopping time $T_{h}$, defined for arbitrary but fixed $h>0$ by

$$
T_{h}:=\inf \left\{t \geq 0: X_{t}=h\right\},
$$


is almost surely finite. Let $\mathcal{F}_{t}$ be the $\sigma$-algebra generated by $\left(X_{s}\right)_{0 \leq s \leq t}$, and consider the stopping time $\sigma$-algebra $\mathcal{F}_{T_{h}}$. Then by the strong Markov property of Lévy processes, see Bertoin [6], Proposition 6 of Chapter I, $\left(X_{T_{h}+t}-X_{T_{h}}\right)_{t \geq 0}$ is independent of $\mathcal{F}_{T_{h}}$ and has the same distribution as $\left(X_{t}\right)_{t \geq 0}$. Writing

$$
\sigma_{\infty}^{2} \stackrel{D}{=} \beta \int_{0}^{\infty} \mathrm{e}^{-X_{t}} \mathrm{~d} t=\beta \int_{0}^{T_{h}} \mathrm{e}^{-X_{t}} \mathrm{~d} t+\beta \int_{T_{h}}^{\infty} \mathrm{e}^{-X_{t}} \mathrm{~d} t=: A_{h}+B_{h}, \quad \text { say, }
$$

we see that $A_{h}$ is $\mathcal{F}_{T_{h}}$-measurable and that

$$
B_{h}=\beta \int_{T_{h}}^{\infty} \mathrm{e}^{-\left(X_{t}-X_{h}\right)} \mathrm{e}^{-X_{T_{h}}} \mathrm{~d} t=\mathrm{e}^{-h} \beta \int_{T_{h}}^{\infty} \mathrm{e}^{-\left(X_{t}-X_{T_{h}}\right)} \mathrm{d} t
$$

is independent of $A_{h}$ and has the same distribution as $\mathrm{e}^{-h} \sigma_{\infty}^{2}$. Thus we have for every $h>0$,

$$
\sigma_{\infty}^{2} \stackrel{D}{=} A_{h}+\mathrm{e}^{-h} \sigma_{\infty}^{2}
$$

with $A_{h}$ and $\sigma_{\infty}^{2}$ being independent, showing that $\sigma_{\infty}^{2}$ is self-decomposable.

The self-decomposability of $\sigma_{\infty}^{2}$ is somewhat surprising, for $\int_{0}^{\infty} \mathrm{e}^{-X_{t}} \mathrm{~d} t$ does not even need to be infinitely divisible for every Lévy process $X_{t}$ tending to $+\infty$ a.s. For example, if $X_{t}=N_{t}+c t, t \geq 0$, with a Poisson process $\left(N_{t}\right)_{t \geq 0}$ and a constant $c>0$, then

$$
0 \leq \int_{0}^{\infty} \mathrm{e}^{-X_{t}} \mathrm{~d} t=\int_{0}^{\infty} \mathrm{e}^{-N_{t}-c t} \mathrm{~d} t \leq \int_{0}^{\infty} \mathrm{e}^{-c t} \mathrm{~d} t=1 / c,
$$

showing that $\int_{0}^{\infty} \mathrm{e}^{-X_{t}} \mathrm{~d} t$ is not infinitely divisible as a bounded non-constant random variable (see Sato [24], Corollary 24.4). This example was constructed by Samorodnitsky [27].

As a self-decomposable distribution, $\sigma_{\infty}^{2}$ has a density, $l$ say. Moreover, if $E L_{1}^{2}<\infty$, then $l$ is infinitely many times differentiable on $(\beta /(-\log \delta), \infty)$ and satisfies the integro-differential equation

$$
\begin{aligned}
& ((-\log \delta) x-\beta) l(x) \\
& =\int_{\beta /(-\log \delta)}^{x} \Pi_{L}\left(\left\{y \in \mathbb{R}:|y|>\sqrt{\left.\left(\frac{x}{v}-1\right) \delta / \lambda\right\}}\right) l(v) \mathrm{d} v, \quad x>\frac{\beta}{-\log \delta} .\right.
\end{aligned}
$$

This follows from Proposition 2.1 of Carmona, Petit and Yor [10]. In Section 5.3 we shall derive another property of $\sigma_{\infty}^{2}$, showing that its distribution has Pareto like tails under suitable conditions.

\subsection{Conditional distributions and tail behaviour of the OU process}

Since the price process $\left(\widetilde{G}_{t}\right)_{t>0}$ in the model of Barndorff-Nielsen and Shephard $[2,3]$ is driven by a Brownian motion independent of the volatility, it 
is not surprising that conditional returns are normally distributed. More precisely, for $t \geq 0, r>0$, let $\widetilde{G}_{t}^{(r)}=\widetilde{G}_{t+r}-\widetilde{G}_{t}$ as in Section 2, and set

$$
\left(\widetilde{\sigma}_{t}^{2 *}\right)^{(r)}:=\int_{t}^{t+r} \widetilde{\sigma}_{s}^{2} \mathrm{~d} s
$$

i.e. the increments of length $r$ of the integrated squared volatility. Then the conditional distribution of $\widetilde{G}_{t}^{(r)}$ given $\left(\widetilde{\sigma}_{t}^{2 *}\right)^{(r)}$ is normal, more precisely

$$
\widetilde{G}_{t}^{(r)} \mid\left(\widetilde{\sigma}_{t}^{2 *}\right)^{(r)} \sim N\left(\mu r+b\left(\widetilde{\sigma}_{t}^{2 *}\right)^{(r)},\left(\widetilde{\sigma}_{t}^{2 *}\right)^{(r)}\right),
$$

see [3], page 170. This is one indication of the fundamental importance of the integrated squared volatility in stochastic volatility models.

For the COGARCH process no easy expression for the returns of the price process is known. However, if $\left(L_{t}\right)_{t \geq 0}$ has Gaussian part $\tau_{L}^{2}$, drift $\gamma_{L, 0}$ and finite Lévy measure coming from a compound Poisson process with jump times $T_{1}<T_{2}<\ldots$ and jump distribution $\rho=\Pi_{L} / \Pi_{L}(\mathbb{R})$, then from $\Delta G_{T_{j}}=$ $\sigma_{T_{j}} \Delta L_{T_{j}}$ follows

$$
\Delta G_{T_{j}} \mid \sigma_{T_{j}} \sim \rho .
$$

For the increments between two jumps, observe that (with $\left(\tau_{L}^{2} W_{s}\right)_{s \geq 0}$ denoting the Brownian motion component of $\left(L_{t}\right)_{t \geq 0}$ )

$$
\begin{aligned}
& G_{T_{j+1}-}-G_{T_{j}-} \\
& =\Delta G_{T_{j}}+G_{T_{j+1}-}-G_{T_{j}} \\
& =\sigma_{T_{j}} \Delta L_{T_{j}}+\gamma_{L, 0} \int_{T_{j}}^{T_{j+1}} \sigma_{s} \mathrm{~d} s+\tau_{L}^{2} \int_{T_{j}}^{T_{j+1}} \sigma_{s} \mathrm{~d} W_{s} .
\end{aligned}
$$

In particular, it can be seen that $G_{T_{j+1}-}-G_{T_{j}-}$, conditioned on $T_{j+1}-T_{j}$, $\sigma_{T_{j}}$ and $\Delta L_{T_{j}}$, is normally distributed.

The tail behaviour of $\widetilde{\sigma}_{\infty}^{2}$ in the OU model depends heavily on the driving Lévy process $\left(L_{t}\right)_{t \geq 0}$. Recall that the Lévy density of $\widetilde{\sigma}_{\infty}^{2}$ and the tail of the Lévy measure of $L_{1}$ are connected by the simple formula (22). Since any positive self-decomposable distribution can occur as $\widetilde{\sigma}_{\infty}^{2}$, this allows for many different tail behaviours. For example, if $k_{+}(x)$ in (21) is chosen to decrease like $x^{-\kappa}$ as $x \rightarrow \infty$ where $\kappa>0$, then $\lim _{x \rightarrow \infty} x^{\kappa} P\left(\widetilde{\sigma}_{\infty}^{2}>x\right)=1 / \kappa$, see Embrechts and Goldie [14] or also Embrechts, Goldie and Veraverbeke [15] in this context. On the other hand, if $\widetilde{\sigma}_{\infty}^{2}$ is generalised inverse Gaussian $\operatorname{GIG}\left(a_{1}, a_{2}, a_{3}\right)$, then it has a probability density given by $f(x)=c x^{a_{1}-1} \exp \left\{-a_{2}^{2} x^{-1} / 2-a_{3}^{2} x / 2\right\}$, $x>0$, with a positive constant $c$ (see, e.g., [3], page 173), so it will not have Pareto like tails unless $a_{3}=0$.

For $\widetilde{G}_{t}$, from (23) it should be expected that the tail behaviour of $\int_{0}^{t} \widetilde{\sigma}_{s}^{2} \mathrm{~d} s$ carries somehow over to the tail behaviour of $\widetilde{G}_{t}$. In order to get insight into the tail behaviour of $\int_{0}^{t} \widetilde{\sigma}_{s}^{2} \mathrm{~d} s$, Barndorff-Nielsen and Shephard [5], equation (31), give a formula for the Lévy density $v$ of $\int_{0}^{t} \widetilde{\sigma}_{s}^{2} \mathrm{~d} s$ in terms of the Lévy 
density of $L_{1}$ (provided $L_{1}$ has a Lévy density; infinite divisibility of the integrated squared volatility can be seen from equation (4) in [5] and the fact that the class of infinitely divisible distributions is closed under convolution and weak convergence). In particular, if either $L_{1}$ or $\widetilde{\sigma}_{\infty}^{2}$ is tempered stable or gamma distributed, it is shown that $v(x)$ behaves asymptotically like $d_{1} x^{-d_{2}} \exp \left\{-d_{3} x\right\}$ as $x \rightarrow \infty$, where $d_{1}, d_{3}>0, d_{2} \in[1,3)$, see [5], Table 3 . In particular, Pareto like tails of $\widetilde{G}_{t}$ are not to be expected in these cases. This is in contrast to the COGARCH process, as will be shown next.

\subsection{Tail behaviour of the COGARCH process}

We now concentrate on the tail behaviour of the COGARCH process, and show that both the tail of the stationary volatility $\sigma_{\infty}$ as well as the tail of $G_{t}$ are Pareto like under weak assumptions, given in terms of the parameters $\delta, \lambda$ and the driving Lévy process $\left(L_{t}\right)_{t \geq 0}$. Recall the notion of $\Psi(\kappa)$ from Lemma 1. Also, for $x \geq 0$, denote $\log ^{+} x=\log (\max \{x, 1\})$. Further, as in Section 4, we assume $\lambda>0$ throughout to avoid a deterministic volatility.

We start with the tail behaviour of $\sigma_{\infty}^{2}$. It can be derived by a simple transformation applied to Lemma 4 of Rivero [26]. For completeness, we shall not deduce it from his result, but rather include a short proof along the lines of $[26]$.

Theorem 6. [Pareto tail behaviour of $\sigma$ ]

Suppose there is $\kappa>0$ such that

$$
E\left|L_{1}\right|^{2 \kappa} \log ^{+}\left|L_{1}\right|<\infty \text { and } \Psi(\kappa)=0 .
$$

Let $\left(\sigma_{t}^{2}\right)_{t \geq 0}$ be the stationary version of the squared volatility process (which exists by Lemma $1(d)$ ). Then there is a constant $C>0$ (which does not depend on $t)$ such that, for any $t \geq 0$,

$$
\lim _{x \rightarrow \infty} x^{\kappa} P\left(\sigma_{t}^{2}>x\right)=C .
$$

Proof. From (7) it is seen that the volatility process $\left(\sigma_{t}^{2}\right)_{t \geq 0}$ satisfies

$$
\sigma_{t}^{2}=\mathrm{e}^{-X_{t-}} \sigma_{0}^{2}+\beta \int_{0}^{t} \mathrm{e}^{X_{s}-X_{t-}} \mathrm{d} s, \quad t>0,
$$

where $\sigma_{0}^{2}$ is independent of $\left(\mathrm{e}^{-X_{t-}}, \beta \int_{0}^{t} \mathrm{e}^{X_{s}-X_{t-}} \mathrm{d} s\right)$ by definition of the COGARCH volatility. Thus (since $\sigma_{0}^{2} \stackrel{D}{=} \sigma_{t}^{2} \stackrel{D}{=} \sigma_{\infty}^{2}$ ) the stationary solution $\sigma_{\infty}^{2}$ satisfies for every $t>0$ the distributional fixed point equation

$$
\sigma_{\infty}^{2} \stackrel{D}{=} M_{t} \sigma_{\infty}^{2}+Q_{t}
$$

where $\sigma_{\infty}^{2}$ is independent of $\left(M_{t}, Q_{t}\right)$ and 


$$
M_{t} \stackrel{D}{=} \mathrm{e}^{-X_{t}}, \quad Q_{t} \stackrel{D}{=} \beta \int_{0}^{t} \mathrm{e}^{-X_{s}} \mathrm{~d} s .
$$

The claim then follows from Theorem 4.1 in Goldie [18], once we have shown that there is some $t>0$ such that

(i) For no $r>0$ is the law of $-X_{t}$ concentrated on $r \mathbb{Z}$

(ii) $E\left|M_{t}\right|^{\kappa}=1$

(iii) $E\left|M_{t}\right|^{\kappa} \log ^{+}\left|M_{t}\right|<\infty$

(iv) $E\left|Q_{t}\right|^{\kappa}<\infty$.

To show $(\mathrm{i})$, recall that $\left(-X_{s}\right)_{s \geq 0}$ is a Lévy process of finite variation with drift $\gamma_{0,-X_{1}}:=\gamma_{0,-X}=\log \delta$, zero Gaussian component and non-zero Lévy measure $\Pi_{-X_{1}}:=\Pi_{-X}$ being concentrated on $(0, \infty)$. The characteristic triplet of the Lévy process $\left(-X_{s}\right)_{s>0}$ is the characteristic triplet of the infinitely divisible distribution $-X_{1}$. For fixed $t$, the characteristic triplet of $-X_{t}$ is $t$ times the characteristic triplet of $-X_{1}$. In particular, the drift and Lévy measure of $-X_{t}$ satisfy $\gamma_{0,-X_{t}}=t \gamma_{0,-X_{1}}$ and $\Pi_{-X_{t}}=t \Pi_{-X_{1}}$. Now let $r>0$. Then $-X_{t}$ is supported on $r \mathbb{Z}$ if and only if $-r^{-1} X_{t}$ is supported on $\mathbb{Z}$, which is equivalent to $-r^{-1} X_{t}$ having drift $\gamma_{0,-r^{-1} X_{t}}$ in $\mathbb{Z}$ and its Lévy measure being supported on $\mathbb{Z}$, see Sato [24], Corollary 24.6. In terms of $-X_{t}$ this is equivalent to $r^{-1} t \log \delta \in \mathbb{Z}$ and $\Pi_{-X_{t}}$ being supported on $r \mathbb{Z}$. Since the supports of the Lévy measures $\Pi_{-X_{1}}$ and $\Pi_{-X_{t}}$ are the same for every $t>0$, but since the drift terms differ by a factor $t$, there cannot exist positive numbers $r_{1}$ and $r_{2}$ such that

$$
\begin{aligned}
& r_{1}^{-1} \log \delta \in \mathbb{Z}, \quad \operatorname{supp}\left(\Pi_{-X_{1}}\right) \subset r_{1} \mathbb{Z}, \quad r_{2}^{-1} \sqrt{2} \log \delta \in \mathbb{Z} \quad \text { and } \\
& \operatorname{supp}\left(\Pi_{-X_{\sqrt{2}}}\right) \subset r_{2} \mathbb{Z} .
\end{aligned}
$$

This gives (i), by chosing $t$ either equal to 1 or to $\sqrt{2}$.

For (ii), note that

$$
E\left|M_{t}\right|^{\kappa}=\exp \left\{\log E \mathrm{e}^{-\kappa X_{t}}\right\}=\exp \{t \Psi(\kappa)\}=1
$$

by assumption. Furthermore, $E \max \left(0,-X_{t}\right) \mathrm{e}^{-\kappa X_{t}}<\infty$ if and only if $\int_{x>1} x \mathrm{e}^{\kappa x} \Pi_{-X}(\mathrm{~d} x)<\infty$, see Sato [24], Theorem 25.3. Using the fact that $\Pi_{X}$ is the image measure of $\Pi_{L}$ under the transformation $\mathbb{R} \rightarrow(-\infty, 0]$, $y \mapsto-\log \left(1+(\lambda / \delta) y^{2}\right)$, this is equivalent to

$$
\int_{|y|>\sqrt{(\mathrm{e}-1) \delta / \lambda}}\left(1+\frac{\lambda}{\delta} y^{2}\right)^{\kappa} \log \left(1+\frac{\lambda}{\delta} y^{2}\right) \Pi_{L}(\mathrm{~d} y)<\infty,
$$

which again is equivalent to $E\left|L_{1}\right|^{2 \kappa} \log ^{+} L_{1}^{2}<\infty$, showing (iii).

From (14) follows $-X_{t} \geq t \log \delta$. Thus $E \mathrm{e}^{-\kappa X_{t}}<\infty$ implies $E \mathrm{e}^{\kappa\left|X_{t}\right|}<\infty$, giving $E \exp \left\{\kappa \sup _{0 \leq s \leq t}\left|X_{s}\right|\right\}<\infty$, see Sato [24], Theorem 25.18. Claim (iv) then follows from

$$
E\left|Q_{t}\right|^{\kappa}=\beta^{\kappa} E\left(\int_{0}^{t} \mathrm{e}^{-X_{s}} \mathrm{~d} s\right)^{\kappa} \leq(\beta t)^{\kappa} E \exp \left\{\kappa \sup _{0 \leq s \leq t}\left|X_{s}\right|\right\}<\infty .
$$


A sufficient condition for (24) to hold is:

Proposition 5. [A sufficient condition] Suppose that (12) holds. Let $D:=\left\{d \in[0, \infty): E\left|L_{1}\right|^{2 d}<\infty\right\}$ and $d_{0}:=$ $\sup D \in[0, \infty]$. Suppose that $d_{0} \notin D$, or that there is $\theta_{0}>0$ such that $0<\Psi\left(\theta_{0}\right)<\infty$. Then (24) holds.

Proof. Suppose $d_{0} \notin D$. Then $d_{0}>0$ and $D$ is an interval containing $[0, \varepsilon)$ for some $\varepsilon>0$. Lemma 1 shows that $\Psi(d)$ is finite for $d \in D$, while $\lim _{d} \gamma_{0} \Psi(d)=$ $\Psi\left(d_{0}\right)=+\infty$. This follows by application of Fatou's Lemma to (17). Choose $\theta_{0} \in\left(0, d_{0}\right)$ such that $\Psi\left(\theta_{0}\right)>0$. Now $\Psi$ is $C^{1}$ on $\left(0, \theta_{0}\right)$, and it follows from (17) that

$$
\Psi^{\prime}(d)=\log \delta+\int_{\mathbb{R}}\left(1+\frac{\lambda}{\delta} y^{2}\right)^{d} \log \left(1+\frac{\lambda}{\delta} y^{2}\right) \Pi_{L}(\mathrm{~d} y)
$$

for $0<d<d_{0}$. Letting $d \searrow 0$, it follows that

$$
\lim _{d \searrow 0} \Psi^{\prime}(d)=\log \delta+\int_{\mathbb{R}} \log \left(1+\frac{\lambda}{\delta} y^{2}\right) \Pi_{L}(\mathrm{~d} y)<0
$$

by (12). Since $\Psi(0)=0$ and $\Psi$ is continuous on $\left[0, \theta_{0}\right)$, it follows that there is $\theta_{1}>0$ such that $\Psi\left(\theta_{1}\right)<0$, and hence there exists $\kappa \in\left(\theta_{1}, \theta_{0}\right)$ such that $\Psi(\kappa)=0$. Since $0<\kappa<\theta_{0}<d_{0}$, finiteness of $E\left|L_{1}\right|^{2 \theta_{0}}$ implies finiteness of $E\left|L_{1}\right|^{2 \kappa} \log ^{+}\left|L_{1}\right|$.

If there is a $\theta_{0}>0$ such that $0<\Psi\left(\theta_{0}\right)<\infty$ then (17) shows that $E\left|L_{1}\right|^{2 \theta_{0}}<\infty$, so $\theta_{0} \in D$. We then find $\kappa>0$ such that $\Psi(\kappa)=0$ as before.

Example 1. (a) Let $0<\delta<1, \lambda>0$, and suppose that (12) holds. Then if all moments of $L_{1}$ exist, or if $\left|L_{1}\right|$ has a Pareto like tail, then $\sigma_{\infty}^{2}$ has Pareto like tail. This follows readily from Proposition 5 and Theorem 6 . For example when $L_{1}$ is generalised inverse Gaussian $\operatorname{GIG}\left(a_{1}, a_{2}, a_{3}\right)$ with $a_{3}>0$ (see Section 5.2), then all moments of $L_{1}$ exist.

(b) Suppose that $E\left|L_{1}\right|^{2 d}<\infty$ for some $d>0$. Then for every $\kappa \in(0, d)$ there exist $\delta_{\kappa} \in(0,1)$ and $\lambda_{\kappa}>0$ such that $\sigma_{\infty}^{2}$ exists and has Pareto like tails. To see this, define

$$
\delta_{\kappa}:=\lambda_{\kappa}:=\exp \left\{-\frac{1}{\kappa} \int_{\mathbb{R}}\left(\left(1+y^{2}\right)^{\kappa}-1\right) \Pi_{L}(\mathrm{~d} y)\right\} .
$$

Then $\delta_{\kappa} \in(0,1)$ and with these parameters, $\Psi(\kappa)=0$. The claim then follows from Theorem 6 .

Our next aim is to show how the Pareto like tail of $\sigma_{\infty}^{2}$ carries over to a Pareto like tail of the distribution of $G_{t}$ for the COGARCH process itself. Before we start proving this, we need the following two lemmas. The first is well known, but for convenience we outline a short proof. Note that no independence assumptions are made. For the definition and properties of regularly varying functions we refer to Bingham et al. [8], or also Feller [17]. 
Lemma 2. Let $Y$ and $Z$ be random variables an a common probability space such that $Y$ has regularly varying right tail with index $-\kappa<0$. Let $d>\kappa$ and suppose that $E|Z|^{d}<\infty$. Then

$$
\lim _{x \rightarrow \infty} \frac{P(Y+Z>x)}{P(Y>x)}=1 .
$$

Proof. $E|Z|^{d}<\infty$ implies $\lim _{x \rightarrow \infty} x^{d^{\prime}} P(|Z|>x)=0$ for every $d^{\prime}<d$, so $\lim _{x \rightarrow \infty} \frac{P(|Z|>x)}{P(Y>x)}=0$. Then $\lim \sup _{x \rightarrow \infty} \frac{P(Y+Z>x)}{P(Y>x)} \leq 1$ follows from

$$
P(Y+Z>x) \leq P(Y>x(1-\varepsilon))+P(Z>x \varepsilon), \quad x>0, \quad \varepsilon>0 .
$$

To show $\liminf _{x \rightarrow \infty} \frac{P(Y+Z>x)}{P(Y>x)} \geq 1$, note that for arbitrary $\varepsilon>0$,

$P(Y+Z>x) \geq P(Y>(1+\varepsilon) x, Z>-\varepsilon x) \geq P(Y>(1+\varepsilon) x)-P(Z \leq-\varepsilon x))$,

so that

$$
\begin{aligned}
& \liminf _{x \rightarrow \infty} \frac{P(Y+Z>x)}{P(Y>x)} \\
& \geq \lim _{n \rightarrow \infty} \frac{P(Y>(1+\varepsilon) x)}{P(Y>x)}-\limsup _{x \rightarrow \infty} \frac{P(Z \leq-\varepsilon x)}{P(Y>x)}=(1+\varepsilon)^{-\kappa} .
\end{aligned}
$$

The following lemma seems intuitively clear. However, its proof requires some technicalities.

Lemma 3. Let $\left(L_{t}\right)_{t \geq 0}$ be a Lévy process of finite variation, and let $X_{t}$ be given by (6). Let $\theta>0$ and $t_{0}>0$. Then $P\left(\int_{0}^{t_{0}} \mathrm{e}^{-\theta X_{s-}} \mathrm{d} L_{s}>0\right)>0$ if and only if $\left(-L_{t}\right)_{t \geq 0}$ is not a subordinator.

Proof. For simplicity in notation we assume $\theta=1$ throughout. It is clear that if $\left(-L_{t}\right)_{t \geq 0}$ is a subordinator, then $P\left(\int_{0}^{t_{0}} \mathrm{e}^{-X_{s-}} \mathrm{d} L_{s}>0\right)=0$, so we only have to prove the converse. So suppose that $\left(L_{t}\right)_{t \geq 0}$, with Lévy measure $\nu$ and drift $\gamma_{0}$, is not the negative of a subordinator. Suppose first that $\nu_{\mid(0, \infty)} \neq 0$. Then there are $0<a<b<\infty$ such that $\nu_{\mid(a, b)}>0$.

Let $t_{0}>0$ be fixed. Let $0<\varepsilon<\min \left\{1 / 2, a, t_{0}\right\}$ and $k \in \mathbb{N}_{0}$. Define the sets $B_{1, \varepsilon}, B_{2, \varepsilon}$ and $B_{3, \varepsilon, k}$ by

$$
\begin{aligned}
& B_{1, \varepsilon}:=\left\{\omega: \sum_{0<s \leq t_{0}-\varepsilon}\left|\Delta L_{s}(\omega)\right|<\varepsilon\right\}, \\
& B_{2, \varepsilon}:=\left\{\omega: \sum_{t_{0}-\varepsilon<s \leq t_{0},\left|\Delta L_{s}(\omega)\right| \leq a}\left|\Delta L_{s}(\omega)\right|<\varepsilon\right\}, \\
& B_{3, \varepsilon, k}:=\left\{\omega: \Delta L_{s}(\omega) \in(a, b) \text { happens for exactly } k \text { values of } s \text { in }\left(t_{0}-\varepsilon, t_{0}\right]\right\} \\
& \cap\left\{\omega: \Delta L_{s}(\omega) \in \mathbb{R} \backslash[-a, b) \text { never happens for } s \text { in }\left(t_{0}-\varepsilon, t_{0}\right]\right\} \text {. }
\end{aligned}
$$


Since $\left(L_{t}\right)_{t \geq 0}$ is of finite variation and $\nu(a, b)>0$, it follows that $P\left(B_{1, \varepsilon}\right)>$ $0, P\left(B_{2, \varepsilon}\right)>0$ and $P\left(B_{3, \varepsilon, k}\right)>0$ (see Sato [24], Theorems 21.9 and 24.10). Moreover, since $\left(L_{s}\right)_{0 \leq s \leq t_{0}-\varepsilon}$ and $\left(L_{s}-L_{t_{0}-\varepsilon}\right)_{s \geq t_{0}-\varepsilon}$ are independent and since for any Lévy process the occurence of large jumps is independent from the occurence of small jumps, it follows that $B_{1, \varepsilon}, B_{2, \varepsilon}$ and $B_{3, \varepsilon, k}$ are all independent. In particular, for $B_{\varepsilon, k}:=B_{1, \varepsilon} \cap B_{2, \varepsilon} \cap B_{3, \varepsilon, k}$ it follows that $P\left(B_{\varepsilon, k}\right)>0$.

From (6) follows, for any $t>0$,

$$
t \log \delta \leq-X_{t} \leq \sum_{0<s \leq t} \log \left(1+\frac{\lambda}{\delta}\left(\Delta L_{s}\right)^{2}\right) .
$$

In particular, on the set $B_{\varepsilon, k}$,

$$
\begin{aligned}
& -X_{t} \leq \frac{\lambda}{\delta} \sum_{0<s \leq t}\left(\Delta L_{s}\right)^{2} \leq \frac{\lambda}{\delta} \sum_{0<s \leq t_{0}-\varepsilon}\left|\Delta L_{s}\right| \leq \frac{\lambda \varepsilon}{\delta} \leq \frac{\lambda}{\delta}, \quad 0 \leq t \leq t_{0}-\varepsilon \\
& -X_{t} \leq \sum_{0<s \leq t} \log \left(1+\frac{\lambda}{\delta}\left(\Delta L_{s}\right)^{2}\right) \leq \frac{\lambda}{\delta}+k \log \left(1+\frac{\lambda}{\delta} b^{2}\right), \quad t_{0}-\varepsilon<t \leq t_{0} .
\end{aligned}
$$

Setting $c_{1}:=\mathrm{e}^{t_{0} \log \delta}$ and $c_{2}:=\mathrm{e}^{\lambda / \delta}$, we obtain for $0<\varepsilon<\min \left\{1 / 2, a, t_{0}\right\}$ and $k \in \mathbb{N}_{0}$ on the set $B_{\varepsilon, k}$,

$$
c_{1} \leq \mathrm{e}^{-X_{s-}} \leq \begin{cases}c_{2}, & \text { for } s \leq t_{0}-\varepsilon, \\ c_{2}\left(1+\frac{\lambda}{\delta} b^{2}\right)^{k}, & \text { for } t_{0}-\varepsilon<s \leq t_{0} .\end{cases}
$$

From this we derive on $B_{\varepsilon, k}$ the estimate

$$
\begin{aligned}
& \int_{0}^{t_{0}} \mathrm{e}^{-X_{s-}} \mathrm{d} L_{s} \\
& =\left(\sum_{0<s \leq t_{0}-\varepsilon}+\sum_{t_{0}-\varepsilon<s \leq t_{0},\left|\Delta L_{s}\right| \leq a}+\sum_{t_{0}-\varepsilon<s \leq t_{0}, \Delta L_{s} \in(a, b)}\right) \mathrm{e}^{-X_{s-} \Delta L_{s}} \\
& \quad+\gamma_{0} \int_{0}^{t_{0}-\varepsilon} \mathrm{e}^{-X_{s-}} \mathrm{d} s+\gamma_{0} \int_{t_{0}-\varepsilon}^{t_{0}} \mathrm{e}^{-X_{s-}} \mathrm{d} s \\
& \geq-c_{2} \varepsilon-c_{2}\left(1+\frac{\lambda}{\delta} b^{2}\right)^{k} \varepsilon+k c_{1} a-\left|\gamma_{0}\right| c_{2} t_{0}-\left|\gamma_{0}\right| c_{2}\left(1+\frac{\lambda}{\delta} b^{2}\right)^{k} \varepsilon
\end{aligned}
$$

Choosing $k$ so large such that $k c_{1} a-\left|\gamma_{0}\right| c_{2} t_{0}>0$ and then $\varepsilon$ sufficiently small, the last estimate will be strictly positive and we obtain for such $\varepsilon$ and $k$ that $\int_{0}^{t_{0}} \mathrm{e}^{-X_{s-}(\omega)} \mathrm{d} L_{s}(\omega)>0$ for $\omega \in B_{\varepsilon, k}$. Since $P\left(B_{\varepsilon, k}\right)>0$, the claim follows for $\nu_{\mid(0, \infty)} \neq 0$.

Now suppose that $\nu_{\mid(0, \infty)}=0$. Since $\left(-L_{t}\right)_{t \geq 0}$ is not a subordinator, the drift $\gamma_{0}$ of $\left(L_{t}\right)_{t \geq 0}$ must be strictly positive. Define the set $D_{\varepsilon, k}$ as 
$\left\{\omega: \sum_{0<s \leq t_{0}}\left|\Delta L_{s}\right|<\varepsilon\right\}$. Then $P\left(D_{\varepsilon, k}\right)>0$, and with $c_{1}$ and $c_{2}$ as before it is the case that, on $D_{\varepsilon, k}$,

$$
\int_{0}^{t_{0}} \mathrm{e}^{-X_{s-}} \mathrm{d} L_{s}=\sum_{0<s \leq t_{0}} \mathrm{e}^{-X_{s-}} \Delta L_{s}+\gamma_{0} \int_{0}^{t_{0}} \mathrm{e}^{-X_{s-}} \mathrm{d} s \geq-c_{2} \varepsilon+\gamma_{0} c_{1} t_{0},
$$

showing that $P\left(\int_{0}^{t_{0}} \mathrm{e}^{-X_{s-}} \mathrm{d} L_{s}>0\right)>0$ when $\varepsilon$ is chosen such that $\varepsilon<$ $\gamma_{0} c_{1} t_{0} / c_{2}$.

The following theorem now gives the Pareto type tail behaviour of $G_{t}$. We need slightly more stringent moment conditions than in Theorem 6 , and assume that the driving Lévy process is of finite variation.

Theorem 7. [Tail behaviour of $G$ ]

Suppose there is $\kappa>0$ and $d>4 \kappa$ such that

$$
E\left|L_{1}\right|^{d}<\infty \text { and } \Psi(\kappa)=0 .
$$

Suppose further that $\left(L_{t}\right)_{t \geq 0}$ is of finite variation. Let $\left(\sigma_{t}^{2}\right)_{t \geq 0}$ be the stationary version of the volatility process, and $G_{t}=\int_{0}^{t} \sigma_{s} \mathrm{~d} L_{s}$ the corresponding COGARCH process. Then if $\left(-L_{t}\right)_{t \geq 0}$ is not a subordinator, for every $t>0$ there exists a positive constant $C_{1, t}$ such that

$$
\lim _{x \rightarrow \infty} x^{2 \kappa} P\left(G_{t}>x\right)=C_{1, t},
$$

and if $\left(-L_{t}\right)_{t \geq 0}$ is a subordinator, then $G_{t} \leq 0$ a.s. Similarly, if $\left(L_{t}\right)_{t \geq 0}$ is not a subordinator, then there exists $C_{2, t}>0$ such that

$$
\lim _{x \rightarrow \infty} x^{2 \kappa} P\left(G_{t} \leq-x\right)=C_{2, t},
$$

and if $\left(L_{t}\right)_{t \geq 0}$ is a subordinator, then $G_{t} \geq 0$ a.s.

Proof. For $0 \leq s \leq t$, define

$$
A_{s}:=\mathrm{e}^{-X_{s-}}, \quad B_{s}:=\beta \int_{0}^{s} \mathrm{e}^{X_{u}-X_{s-}} \mathrm{d} u .
$$

Then from (7)

$$
\sigma_{s}=\sqrt{A_{s} \sigma_{0}^{2}+B_{s}}=\sqrt{A_{s}} \sigma_{0}+\frac{B_{s}}{\sqrt{A_{s} \sigma_{0}^{2}+B_{s}}+\sqrt{A_{s} \sigma_{0}^{2}}} .
$$

Defining

$$
\begin{aligned}
Y_{t} & :=\sigma_{0} \int_{0}^{t} \sqrt{A_{s}} \mathrm{~d} L_{s}, \quad \zeta_{s}:=\frac{B_{s}}{\sqrt{A_{s} \sigma_{0}^{2}+B_{s}}+\sqrt{A_{s} \sigma_{0}^{2}}}, \quad \text { and } \\
Z_{t} & :=\int_{0}^{t} \zeta_{s} \mathrm{~d} L_{s},
\end{aligned}
$$


we obtain

$$
G_{t}=\int_{0}^{t} \sigma_{s} \mathrm{~d} L_{s}=Y_{t}+Z_{t}, \quad t>0
$$

From Theorem 6 we know that $\lim _{x \rightarrow \infty} x^{2 \kappa} P\left(\sigma_{0}>x\right)=C$ for some positive constant $C$. Suppose we show that there is an $d^{\prime}>2 \kappa$ such that $E\left|\int_{0}^{t} \sqrt{A_{s}} \mathrm{~d} L_{s}\right|^{d^{\prime}}<\infty$. Then a result of Breiman [9], using the independence of $\sigma_{0}$ and $\int_{0}^{t} \sqrt{A_{s}} \mathrm{~d} L_{s}$, yields the existence of strictly positive constants $C_{1, t}, C_{2, t}$ such that

$$
\lim _{x \rightarrow \infty} x^{2 \kappa} P\left(Y_{t}>x\right)=C_{1, t}, \quad \lim _{x \rightarrow \infty} x^{2 \kappa} P\left(Y_{t} \leq-x\right)=C_{2, t},
$$

provided $P\left(\int_{0}^{t} \sqrt{A_{s}} \mathrm{~d} L_{s}>0\right)>0$ and $P\left(\int_{0}^{t} \sqrt{A_{s}} \mathrm{~d} L_{s}<0\right)>0$, respectively. We shall verify the required moment condition with $d^{\prime}:=d / 2$. Note that

$$
\left|\int_{0}^{t} \sqrt{A_{s}} \mathrm{~d} L_{s}\right| \leq \sup _{0 \leq s \leq t} \mathrm{e}^{-X_{s} / 2}\left\|L_{t}\right\|_{\mathrm{TV}}
$$

where $\left\|L_{t}\right\|_{\mathrm{TV}}$ denotes the total variation of $\left(L_{s}\right)_{0 \leq s \leq t}$ on $[0, t]$, and we also have that $E \sup _{0 \leq s \leq t} \mathrm{e}^{-d^{\prime} X_{s}}<\infty$ since $E \mathrm{e}^{-d^{\prime} X_{1}}<\infty$ (as in the proof of Theorem 6), and that $E\left\|L_{t}\right\|_{\mathrm{TV}}^{2 d^{\prime}}$ is finite since $E\left|L_{1}\right|^{2 d^{\prime}}$ is finite by assumption (see Sato [24], Theorem 21.9); also, it follows from Hölder's inequality that

$$
E\left(\sup _{0 \leq s \leq t} \mathrm{e}^{-X_{s} / 2}\left\|L_{t}\right\|_{\mathrm{TV}}\right)^{d^{\prime}} \leq\left(E \sup _{0 \leq s \leq t} \mathrm{e}^{-d^{\prime} X_{s}}\right)^{1 / 2}\left(E\left\|L_{t}\right\|_{\mathrm{TV}}^{2 d^{\prime}}\right)^{1 / 2}<\infty .
$$

So the moment condition is established, with $d^{\prime}=d / 2>2 \kappa$.

To get an estimate for $Z_{t}$, note that $X_{u} \leq-u \log \delta$ by (6), so that for $0 \leq s \leq t$

$$
\zeta_{s} \leq \sqrt{B_{s}}=\sqrt{\beta} \sqrt{A_{s}} \sqrt{\int_{0}^{s} \mathrm{e}^{X_{u}} \mathrm{~d} u} \leq \sqrt{\beta} \sqrt{t \delta^{-t}} \sqrt{A_{s}} .
$$

This implies, with $d^{\prime}$ as above,

$$
E\left|Z_{t}\right|^{d^{\prime}} \leq \beta^{d^{\prime} / 2} t^{d^{\prime} / 2} \delta^{-d^{\prime} t / 2} E\left|\sup _{0 \leq s \leq t} \mathrm{e}^{-X_{s} / 2}\left\|L_{t}\right\|_{\mathrm{TV}}\right|^{d^{\prime}}<\infty
$$

as already shown. Now if $P\left(\int_{0}^{t} \sqrt{A_{s}} \mathrm{~d} L_{s}>0\right)>0$, i.e. $\left(-L_{t}\right)_{t \geq 0}$ is not a subordinator by Lemma 3, an application of Lemma 2 to (27) gives the result. On the other hand, if $P\left(\int_{0}^{t} \sqrt{A_{s}} \mathrm{~d} L_{s}>0\right)=0$, i.e. if $\left(-L_{t}\right)_{t \geq 0}$ is a subordinator, then also $G_{t}=\int_{0}^{t} \sigma_{s} \mathrm{~d} L_{s} \leq 0$ a.s. The assertion for the left tail behaviour of $G_{t}$ follows similarly. 
Examples for the application of Theorem 7, similar to Example 1(a) in the case when all moments of $L_{1}$ exist, or Example 1(b) can be easily stated. We conclude this section with the observation that with the same methods of proof the tail behaviour of the integrated squared volatility can be determined. Here, a weaker moment condition is sufficient:

Proposition 6. [Tail behaviour of the integrated squared volatility] Let the conditions of Theorem 6 be satisfied. In addition assume that there is $d>2 \kappa$ such that $E\left|L_{1}\right|^{d}<\infty$. Let $\left(\sigma_{t}^{2}\right)_{t \geq 0}$ be the stationary version. Then, for any $t>0$ there is a constant $C_{t}>0$ such that

$$
\lim _{x \rightarrow \infty} x^{\kappa} P\left(\int_{0}^{t} \sigma_{s}^{2} \mathrm{~d} s>x\right)=C_{t}
$$

\section{Conclusion}

We have compared the probabilistic properties of both the stochastic volatility model of Barndorff-Nielsen and Shephard and the COGARCH process. Both volatility models are positive Markov processes, which exhibit jumps and decrease exponentially between jumps. Although the log price process is defined in terms of an independent Brownian motion for the OU model and in terms of the same driving Lévy process for the COGARCH process, the autocorrelation structure of the returns is similar for both processes. Furthermore, we have seen that the tail behaviour in the OU model depends heavily on the driving Lévy process, while for the COGARCH model Pareto like tails occur in most cases under weak regularity conditions.

\section{Acknowledgements}

We thank Marc Yor and Victor Rivero for interesting discussions and their considerable efforts concerning the tail behaviour of the COGARCH model. Further, we thank Gennady Samorodnitsky for answering the question if the stationary distribution of the COGARCH process is self-decomposable, and for his generosity in allowing us to include this result in Theorem 5 .

Thanks also to Ole Barndorff-Nielsen for helpful comments on the paper, and in particular for drawing our attention to the quadratic variation of the COGARCH process, which led to Proposition 3.

Parts of this research were carried out while A. Lindner was visiting the Centre for Mathematical Analysis and the School of Finance \& Applied Statistics at ANU in Canberra. He takes pleasure in thanking both for their hospitality. This research was partially supported by ARC grant DP0210572. A. Lindner was supported by the German Science Foundation (Deutsche Forschungsgemeinschaft). 


\section{References}

1. Barndorff-Nielsen, O.E.: Superposition of Ornstein-Uhlenbeck type processes. Theory Probab. Appl. 45, 175-194 (2001)

2. Barndorff-Nielsen, O.E., Shephard, N.: Modelling by Lévy processes for financial econometrics. In: O.E. Barndorff-Nielsen, T. Mikosch, S. Resnick (Eds.), Lévy processes, theory and applications, pp. 283-318. Boston: Birkhäuser 2001

3. Barndorff-Nielsen, O.E., Shephard, N.: Non-Gaussian Ornstein-Uhlenbeckbased models and some of their uses in financial economics (with discussion). J. R. Statist. Soc. Ser. B 63, 167-241 (2001)

4. Barndorff-Nielsen, O.E., Shephard, N.: Econometric analysis of realised volatility and its use in estimating stochastic volatility models. J. R. Statis. Soc. Ser. B 64, 253-280 (2002)

5. Barndorff-Nielsen, O.E., Shephard, N.: Integrated OU processes and nonGaussian OU-based stochastic volatility models. Scand. J. Statist. 30, 277-295 (2003)

6. Bertoin, J.: Lévy processes. Cambridge: Cambridge University Press 1996

7. Bertoin, J., Yor, M.: On the entire moments of self-similar Markov processes and exponential functionals of Lévy processes. Ann. Fac. Sci. Toulouse Math. (6) 11, 33-45 (2002)

8. Bingham, N.H., Goldie, C.M., Teugels, J.L.: Regular variation. Cambridge: Cambridge University Press 1987

9. Breiman, L.: On some limit theorems similar to the arc-sine law. Theory Probab. Appl. 10, 323-331 (1965)

10. Carmona, P., Petit, F., Yor, M.: On the distribution and asympotic results for exponential functionals of Lévy processes. In: M. Yor (Ed.), Exponential functionals and principal values related to Brownian motion, pp. 73-121. Madrid: Biblioteca de le Revista Matemàtica Iberoamericana 1997

11. Carmona, P., Petit, F., Yor, M.: Exponential functionals of Lévy processes. In: O.E. Barndorff-Nielsen, T. Mikosch, S. Resnick (Eds.), Lévy processes, theory and applications, pp. 41-55. Boston: Birkhäuser 2001

12. Duan, J.C.: Augmented $\operatorname{GARCH}(p, q)$ process and its diffusion limit. J. of Econometrics 79, 97-127 (1997)

13. Engle, R.F.: ARCH: selected readings. Oxford: Oxford University Press 1995

14. Embrechts, P., Goldie, C.M.: On convolution tails. Stoch. Proc. Appl. 13, 263278 (1982)

15. Embrechts, P., Goldie, C.M., Veraverbeke, N.: Subexponentiality and infinite divisibility. Zeit. Wahrsch. Verw. Gebiete 49, 335-347 (1979)

16. Erickson, K.B., Maller, R.A.: Generalised Ornstein-Uhlenbeck processes and the convergence of Lévy integrals. Séminaire des Probabilités, to appear (2004)

17. Feller, W.: An introduction to probability theory and its applications II. New York: Wiley 1971

18. Goldie, C.M.: Implicit renewal theory and tails of solutions of random equations. Ann. Appl. Probab. 1(1), 126-166 (1991)

19. Jacod, J. and Shiryaev, A.N.: Limit theorems for stochastic processes. 2nd edn. Heidelberg: Springer 2003

20. Jeantheau, T.: A link between complete models with stochastic volatility and ARCH models. Finance Stochast. 8, 111-131 (2004)

21. Kim, S., Shephard, N., Chib, S.: Stochastic volatility: likelihood inference and comparison with ARCH models. Review of Economic Studies 65, 361-393 (1998) 
22. Klüppelberg, C., Lindner, A., Maller, R.: A continuous time GARCH process driven by a Lévy process: stationarity and second order behaviour. J. Appl. Probab. 41(3) (to appear) (2004)

23. Nelson, D.B.: ARCH models as diffusion approximations. J. of Econometrics 45, 7-38 (1990)

24. Sato, K.-I.: Lévy processes and infinitely divisible distributions. Cambridge: Cambridge University Press 1999

25. Shephard, N.: Stochastic volatility: selected readings. Oxford: Oxford University Press 2004

26. Rivero, V.: Recurrent extensions of self-similar Markov processes and Cramér's condition. Bernoulli (to appear). Available at http://www.proba.jussieu.fr/mathdoc/preprints, Prépublication no. 838.

27. Samorodnitsky, G.: Private communication (2004)

28. Wang, Y.: Asymptotic nonequivalence of GARCH models and diffusions. Ann. Statist. 30, 754-783 (2002) 